\title{
A Real-time Stitching Algorithm for UAV Aerial Images
}

\author{
Peng Xiong, Xianpeng Liu, Chao Gao, Zan Zhou, Chunxiao Gao, Qiongxin Liu \\ School of Computer Science \\ Beijing Institute of Technology \\ Beijing, China \\ bychance@gmail.com
}

\begin{abstract}
Real-time stitching is a research focus of UAV area. The speed and accuracy of stitching algorithm are both important for commanders to make right decisions in battlefield. In this paper, a real-time UAV aerial image stitching algorithm based on predicted region matching is proposed. A new matching strategy is applied to the feature matching step. The transformation matrix will be calculated by the matches between features in current image and features in a predicted region. Experimental results show that, comparing with traditional method, the new algorithm can reduce much stitching errors while keeping the process time almost the same.
\end{abstract}

Keywords- real-time stitching; predicted region; UAV; aerial image

\section{INTRODUCTION}

UAV aerial images stitching is an important technology in modern battlefield. A UAV flies in Z-shape order when it investigates a rectangular area. So the aerial image set can be divided to some image sets, the UAV flies in a straight line in every image set.

There're mainly two kinds of image registration method: 2D method based on sequential processing and 2D transformations, and 3D method based on global processing and camera model[1]. 2D method is widely used in normal photo stitching because of its high speed[2]. 3D method is used in $\mathrm{SfM}$ (Structure from Motion) because it can handle a large number of images and get the highest accuracy[3]. However, these traditional methods cannot meet the requirements of aerial images stitching. Huge errors will be introduced in the overlapping area between two adjacent image sets when 2D method is applied. The speed of 3D method is too slow to be applied in real-time investigation. Moreover, the computational complexity of $3 \mathrm{D}$ method is not linear, so $2 \mathrm{D}$ method is a better choice for real-time stitching.

In order to reduce the error of traditional 2D method, many researchers have done a lot of hard work. WANG Shuming uses ground control points to assist image registration[4]. However, it's hard to put ground control points in the battlefield. McCartney uses a new strategy: every image set is stitched separately, then stitch all the small result images to a large one[3]. However, it will be impossible to stitch two result images if every set has a large number of images.

In this paper, a real-time $2 \mathrm{D}$ stitching algorithm is proposed based on the characteristic of UAV aerial images. The transformation matrix will be calculated by the matches between features in current image and features in a predicted region. The predicted region will be updated after the actual registration position of current image is decided. This algorithm keeps the balance of speed and accuracy.

\section{ALGORITHM WORKFLOW}

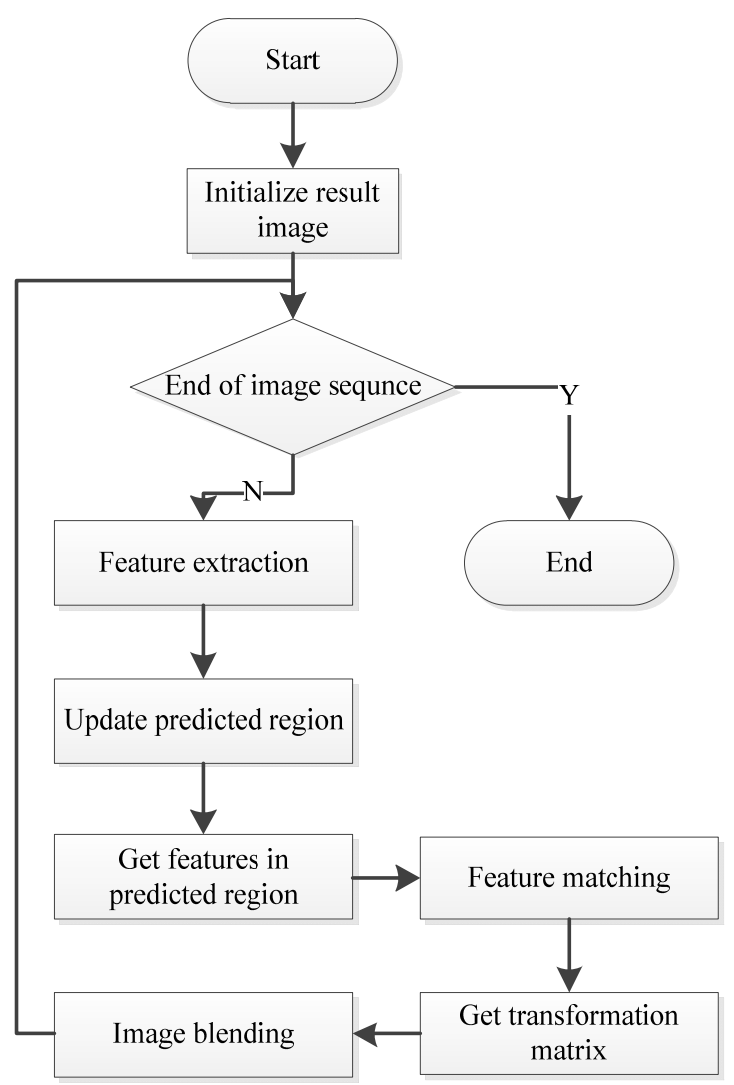

Figure 1. Workflow chart of algorithm

The proposed algorithm is divided into different steps. Process every image in aerial image sequence following these steps in Fig. 1.

Setp1 is feature extraction of current image. SURF is applied to this step because of its fast speed and invariant to illumination[5]. Step2 is updating predicted region. It is the most important step to improve stitching quality. Step3 is putting all features within the predicted region into a set. Step4 is feature matching between the feature set of current 
image and the feature set of predicted region. K-D Tree is applied for pairwise matching. Step5 is calculation of affine transformation matrix based on RANSAC[6]. Step6 is image blending based on feather blending algorithm to reduce ghosting in overlapping area after placing current image in result image.

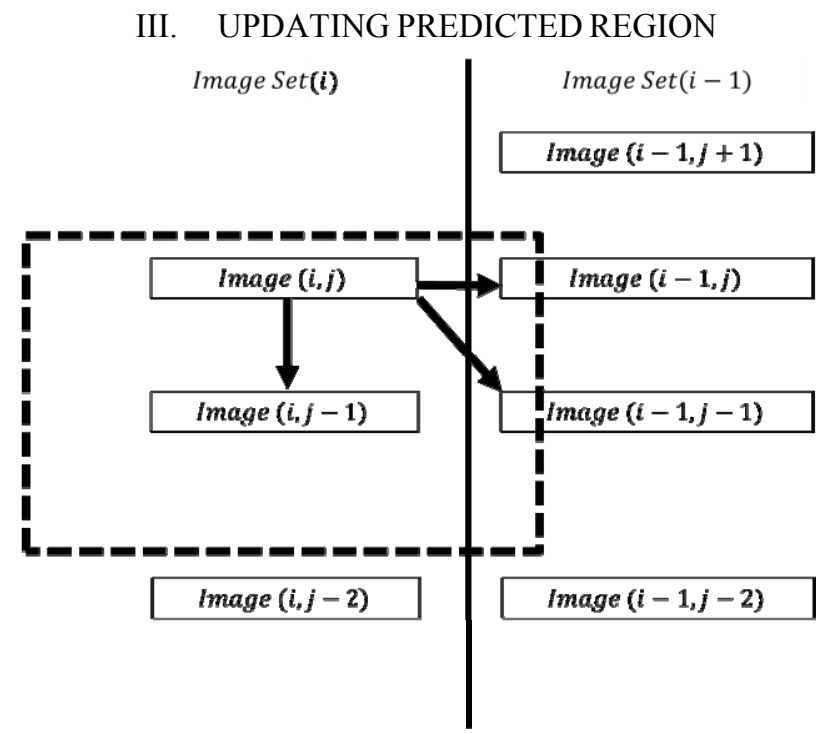

Figure 2. Predicted region contains images both from current and previous image sets. Arrows stand for calculation dependency

In this paper, $i$-th image set is marked as Image Set(i). $j$ th image in Image Set(i) is marked as Image $(i, j)$. In order to make the algorithm description simple, it's assumed that every image set has the same number of images, and the indexes of images follow two-dimension array order, the first image is Image $(0,0)$.

In traditional 2D stitching method, only the information from Image( $i, j-1)$ will be used to stitch Image( $i, j)$, when $j>0$. This approach brings huge accumulated error when the image sequence has large number of images. So the seam of overlapping area between image sets can be observed easily. The images in the last part of image sequence are hardly stitched to result image due to accumulated error.

In the algorithm this paper proposed, Image $(i, j)$ will be stitched according to all the information in the predicted region. For example, in Fig. 2, all the features from Image $(i, j-1)$, Image( $i-1, j)$ and Image $(i-1, j-1)$ within the predicted region will be taken into consideration. So the transformation matrix in Step5 can meet the right position requirements for Image( $i, j-1)$, Image( $i-1, j)$ and Image( $i-1, j-1)$ at the same time. The accumulated error will be reduced.

Generally speaking, if the size of predicted region is too large, it will slow down the speed and lead to mismatch. On the other hand, if the size is too small, some important information from surrounding images will be missing.

Overlap ratio $\varepsilon$ is introduced here. It's defined by the percentage of overlapping between two adjacent images. $\varepsilon$ comes from the UAV aerial image sequence. $\varepsilon$ is assumed to be a constant in all images from the same image sequence.
The current flight direction cannot be predicted directly after stitching its previous image. The predicted region can be decided by the position of previous image and $\varepsilon$.

Define $R_{0}$ as the minimum bounding rectangle of the previous image, $P_{0}$ is the center point of $R_{0}, S_{0}$ is the size of $R_{0}$.

$$
S_{0}=W \times H
$$

Define $R_{1}$ as the predicted region rectangle, $P_{1}$ is the center point of $R_{1}, S_{1}$ is the size of $R_{1}$.

The relationship between $R_{0}$ and $R_{1}$ is shown in Fig. 3 .

The calculation of $P_{1}$ and $S_{1}$ follows the equations below.

$$
S_{1}=[2(1-\varepsilon)+1] W \times[2(1-\varepsilon)+1] H
$$

$$
P_{1}=P_{0}
$$

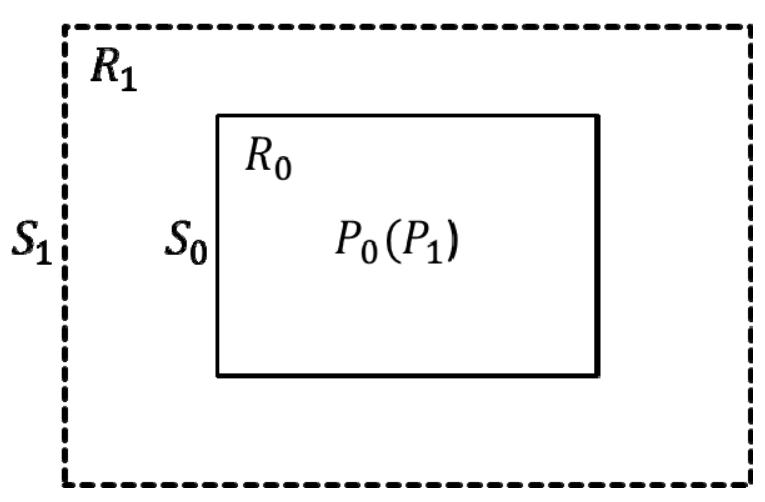

Figure 3. Calculation of predicted region

\section{ERROR ANALYSIS}

Accumulated error increases in stitching process of every image. It is assumed that every image brings the same amount of accumulated error. So the total amount of accumulated error of Image $(i, j)$ is decided by the calculation distance from Image $(0,0)$ to Image $(i, j)$.

Define $E_{0}(i, j)$ as the accumulated error of Image $(i, j)$ when traditional 2D stitching algorithm is applied. The transformation matrix is calculated by the previous image, so $E_{0}(i, j)$ will follow the equation below.

$$
E_{0}(i, j)=i * n+j
$$
1)

There're similar equations for Image $(i-1, j)$ and Image $(i, j-$

$$
\begin{gathered}
E_{0}(i-1, j)=(i-1) * n+j \\
E_{0}(i, j-1)=i * n+j-1
\end{gathered}
$$


Define $E_{1}(i, j)$ as the accumulated error of $\operatorname{Image}(i, j)$ when the new stitching algorithm based on predicated region matching is applied. $E_{1}(i, j)$ should be a combination of $E_{0}(i-$ $1, j)$ and $E_{0}(i, j-1)$ :

$$
E_{1}(i, j)=f \times E_{0}(i-1, j)+g \times E_{0}(i, j-1)
$$

In equation (7), $f$ and $g$ are real numbers in range $[0,1)$, standing for the weight of combination. The distance will be reduced.

From the analysis above, we can get the relationship:

$$
E_{1}(i, j)<E_{0}(i, j)
$$

So the accumulated error in new algorithm is less than traditional algorithm.

\section{EXPERIMENTS}

The experiments run in a PC with Intel Core i7-2600, 8G RAM and Visual Studio 2010 Express. Two UAV aerial image sequences are chosen to validate the efficiency of our new algorithm. Dataset A comes from Google Earth; it contains 2 image sets and 50 images in total. Dataset B comes from photos which are captured from camera mounted in UAV; it contains 4 image sets and 100 images in total. Working resolution is $0.6 \mathrm{M}$ pixel. Traditional $2 \mathrm{D}$ stitching algorithm and our new algorithm based on predicted region matching are applied in order to compare the quality. 3D stitching algorithm is also applied to these experiments. The result images from 3D algorithm are used as standard images because of its best quality. Experimental results are shown in Fig. 4 - Fig. 6 and Tab. 1.

TABLE I. EXPERIMENT DATA

\begin{tabular}{|l|l|l|l|}
\hline \multirow{2}{*}{ Dataset } & \multicolumn{3}{|c|}{ Performance } \\
\cline { 2 - 4 } & \multicolumn{1}{|c|}{ Algorithm } & Time(s) & Error(pixel) \\
\hline \multirow{3}{*}{ A } & Tranditional 2D & 25 & $1.21 \times 10^{5}$ \\
\cline { 2 - 4 } & Predicted Region Matching & 26 & $4.35 \times 10^{4}$ \\
\hline \multirow{3}{*}{ B } & Tranditional 2D & 135 & $9.62 \times 10^{7}$ \\
\cline { 2 - 4 } & Predicted Region Matching & 149 & $1.96 \times 10^{6}$ \\
\hline
\end{tabular}

Tab. 1 compares the performance for two algorithms. Processing time only increases a little in predicted region matching algorithm. Result images from 3D stitching algorithm are used as standard images. Error of a feautre point is the euclidean distance(in pixel) between the position of one feature point in $3 \mathrm{D}$ result image and the position of the same feature point in other result image. The total error is the sum of error of all feature points. The total error is reduced a lot of our new algorithm comparing with traditional 2D stitching algorithm.

Fig. 4 shows a same part(around image 13 and image 28) of different results image in dataset $\mathrm{A}$. The bottom part of the mountain is mislocated in (a). The stitching seam is very obvious. (b) and (c) are almost the same.

Fig. 5 shows a same part(around image 27 and image 32) of different results image in dataset B. The roads in the upper right are misplaced in (a). The position in (b) is right while the illumination is a little different from the surroundings.

Fig. 6 shows a same part(around image 56 and image 80) of different results image in dataset B. Accumulated error is very large when stitching Image Set(4) in (a). The left part is totally mislocated, the road is cut off. (b) is very close to (c) except for the upper right part of the lake.

All the experiment results show the superiority of the propsed algoithm based on predicted region matching in this paper compaed to traditional 2D stitching algorithm. It reduces accumulated error and gives good quality result image while the processing time has only a little increase.

\section{CONCLUSION}

A new stitching algorithm is proposed in this paper which overcomes the shortcoming of traditional 2D stitching algorithm. The transformation matrix is calculated between features in one image and all previous features in its predicted position. It will make the transformation fit all surrounding images. Accumulated error will be reduced a lot while the processing time keeps almost the same. This algorithm meets the real-time and accuracy requirements of UAV investigation in battlefield.

However, it still needs improvements. In step3, if the number of features in previous images is much more than the number of features in previous image set due to high overlapping ratio between images in same image set, the transformation will fit previous image better according to RANSAC algorithm. We will consider how to choose features in step2 to solve this problem.

\section{REFERENCES}

[1] R. Szeliski, "Image Alignment and Stitching: A Tutorial," Foundations and Trends ${ }^{\circledR}$ in Computer Graphics and Vision, vol. 2, no. 1, pp. 1-104, 2006.

[2] M. I. McCartney, S. Zein-Sabatto, and M. Malkani, "Image registration for sequence of visual images captured by UAV," in Computational Intelligence for Multimedia Signal and Vision Processing, 2009. CIMSVP'09. IEEE Symposium on, 2009, pp. 9197.

[3] J. Jia and C. Tang, "Image stitching using structure deformation," IEEE Transactions on Pattern Analysis and Machine Intelligence PAMI, vol. 30, no. 4, pp. 617-631, 2008.

[4] Shuming Wang, Aiwu Zhang and Yingying Cui, "Unmanned Airshipbased Digital Photogrammetry System and Aerial Sequnce Image Stitching" Science of Surverying and Mapping, vol. 35, no. S1, pp. 81-83, 2010.

[5] L. Juan and O. Gwun, "A comparison of sift, pca-sift and surf," International Journal of Image Processing (IJIP), vol. 3, no. 4, pp. 143-152, 2009.

[6] A. Cartography and M. Park, "Random Sample Consensus : A Paradigm for Model Fitting with," Communications of the ACM, vol. 24, no. 6, pp. 381-395, 1981. 


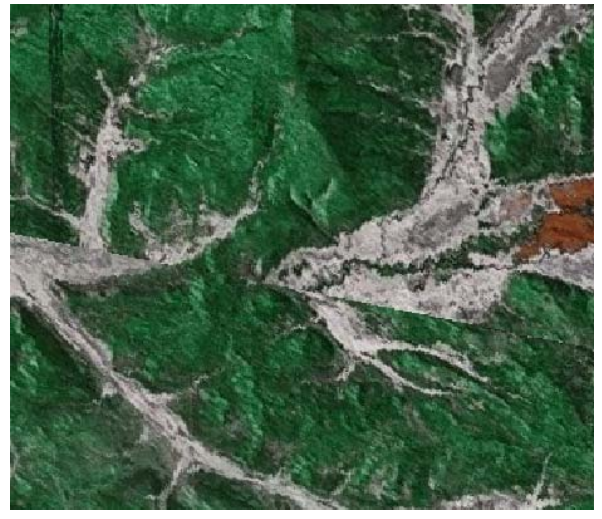

(a) Traditional 2D

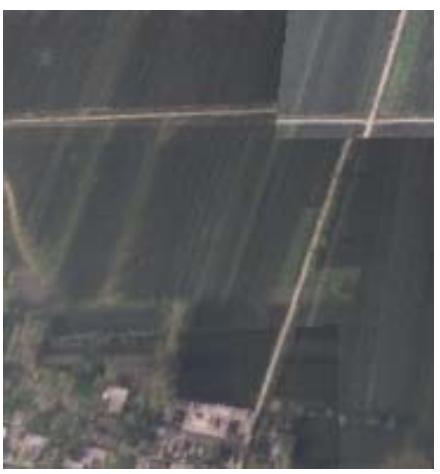

(a) Traditional 2D

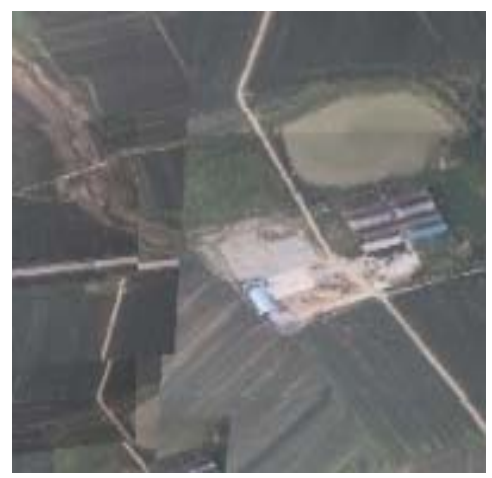

(a) Traditional 2D

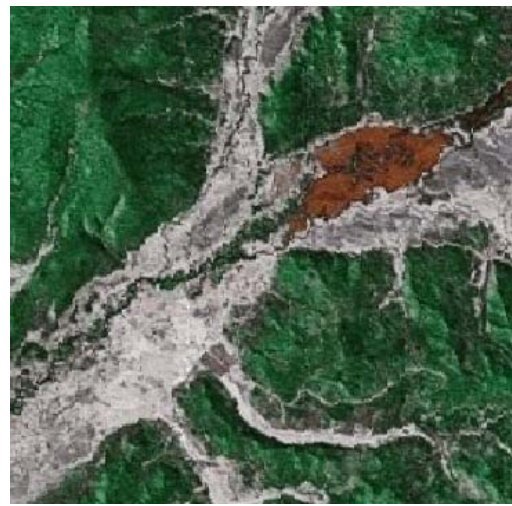

(b) Predicted Region Matching

Figure 4. Part of result images of dataset A

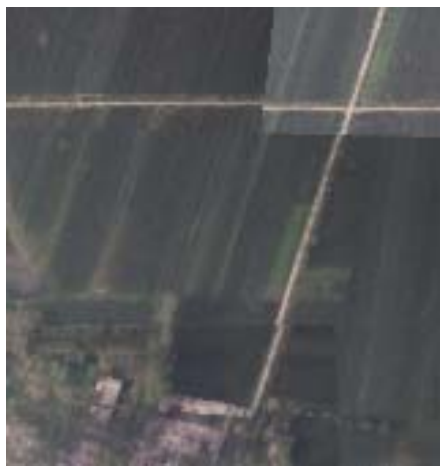

(b) Predicted Region Matching

Figure 5. Part of result images of dataset $B$

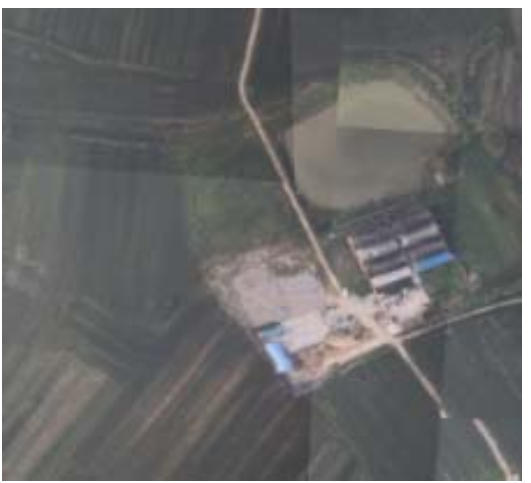

(b) Predicted Region Matching

Figure 6. Part of result images of dataset B

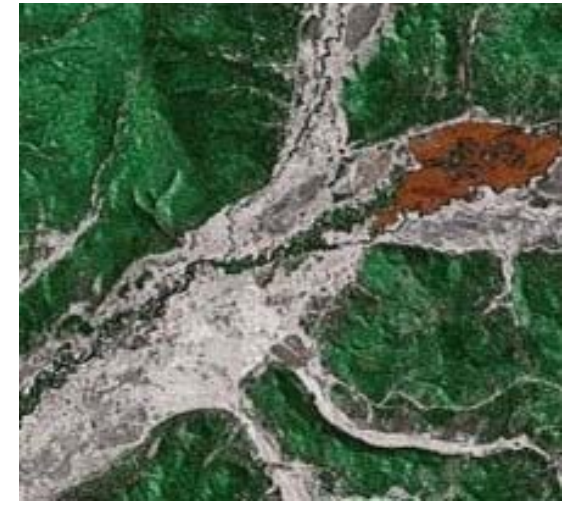

(c) Traditional 3D

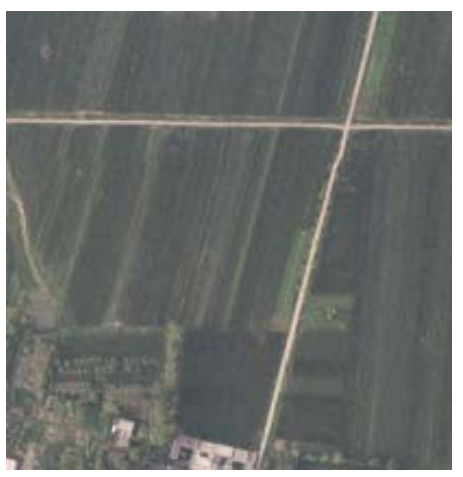

(c) Traditional 3D

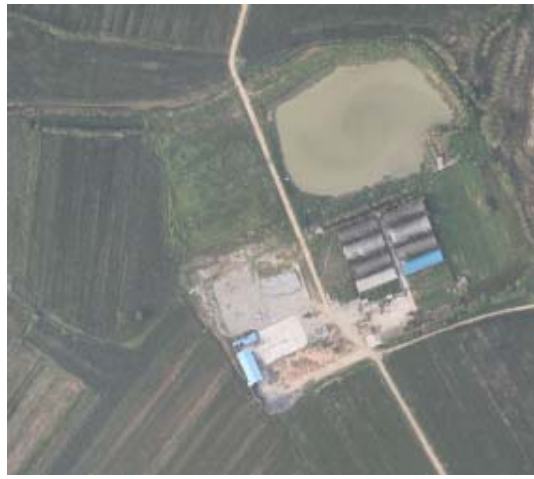

(c) Traditional 3D 